\title{
Estudio sobre la Calidad de la Educación en Escuelas de Barrios Periféricos de Santiago de Chile: ¿Una Justicia ante la Marginalización Social?
}

\author{
Study on the Quality of Education in Schools of Peripheral \\ Neighborhoods of Santiago de Chile: A Justice in the Face of \\ Social Marginalization?
}

\author{
Ignacio E. Reyes * \\ Universidad de Playa Ancha, Chile
}

\begin{abstract}
Las reformas escolares del país han puesto especial importancia en la calidad de los resultados estandarizados que miden los aprendizajes de los alumnos. La medición del rendimiento aparece como la puerta de acceso al progreso. La siguiente contribución trata sobre la escolarización de niños de tres comunas periféricas de la Región Metropolitana de Santiago. En particular, abordaremos los puntos de vista de profesores de escuelas municipales y particulares subvencionadas. La vida en una población de la zona sur evidencia de manera fehaciente lo que el sociólogo peruano Aníbal Quijano llamó el polo marginal y/o exclusión social. Este estudio busca reflexionar sobre la calidad de la educación, a la luz de la experiencia de las escuelas en barrios periféricos. Asimismo, observar diferencias entre escuelas municipales y particulares subvencionadas. Para esto nos concentraremos en el posicionamiento sobre la prueba SIMCE. La justicia social aparece como la entrada al progreso en base a la calidad de la educación y meritocracia individual; por otro una justicia de corto plazo que se traduce en asumir las condiciones de pobreza de los alumnos y entregar un apoyo socio-afectivo.
\end{abstract}

Descriptores: Enseñanza pública; Enseñanza privada; Rendimiento escolar; suburbios; Exclusión social.

The country's school reforms have placed special importance on the quality of the standardized results that measure student learning. Performance measurement appears as the gateway to progress. The following contribution deals with the schooling of children in three peripheral communities of the Santiago Metropolitan Region. In particular, we will address the views of teachers from municipal and private subsidized schools. Life in a southern zone population is evidence of what the Peruvian sociologist Aníbal Quijano called the marginal pole and/or social exclusion. This study seeks to reflect on the quality of education, in light of the experience of schools in peripheral neighborhoods. Also, to observe differences between municipal schools and subsidized private schools. For this we will concentrate on the positioning on the SIMCE test. Social justice appears as the entrance to progress based on the quality of education and individual meritocracy; on the other hand, a short-term justice that translates into assuming the conditions of poverty of students and providing socio-affective support.

Keywords: Public education; Private education; Academic achievement; Suburbs; Social exclusion.

*Contacto: ignacio.reyes.cayul@gmail.com

ISSN: 2254-3139

www.rinace.net/riejs/

revistas.uam.es/riejs
Recibido: $\quad 15$ de abril 2020

$1^{\text {a }}$ Evaluación: 5 de junio 2020

$2^{\text {a }}$ Evaluación: 9 de septiembre 2020

Aceptado: 26 de septiembre 2020 


\section{Introducción}

Hablar del polo marginal ${ }^{1}$ se inscribe en la llamada heterogeneidad estructural, esto es, un sistema con característica de total o global que construye una diversidad de experiencias de vida. La marginalización caracteriza la vida de los barrios periféricos. Esta diversidad evidencia un sinfín de relaciones con la sociedad imperante. Así, el desempleo, la pobreza, la violencia, el narcotráfico, la falta de oportunidades y movilidad social. Estas condiciones son, no un estado coyuntural que será superado por el progreso inevitable de la modernidad, sino que son perennes para un vasto número de sujetos. La escuela no queda fuera del polo marginal de la economía: navega entre el sueño de una vida con mejores oportunidades y contener a niños y familias del desempleo de larga duración, comercio infernal, zonas de narcotráfico y balaceras.

Las reformas escolares del país han puesto especial importancia en la calidad de los resultados estandarizados que miden los aprendizajes de los alumnos. La medición del rendimiento aparece como la puerta de acceso al progreso. No obstante, este proyecto, las escuelas ubicadas en la periferia deben verse enfrentadas a imperativos cotidianos que le ubican como una zona de protección socio-afectiva con los alumnos. Así, el papel de protectora de la infancia se combina con su rol de inclusión a la sociedad a quienes están tradicionalmente fuera de la actividad económica del país.

Por otro lado, la expansión de la matrícula nacional ha sido planteada desde la apertura de escuelas particulares con subvención estatal más la administración municipal de la oferta pública. La presencia de escuelas privadas en zonas periféricas constituye una novedad en el clásico rol de la escuela privada. Apareciendo comportamientos nuevos para estos sectores, tales como la elección de la escuela por parte de los apoderados. Constituyendo, por ende, un verdadero cruce de fronteras entre lo público y lo privado.

Tomando en cuenta estos antecedentes, nuestra investigación se centra en los discursos de profesores de escuelas particulares subvencionadas y municipales. Mediante entrevistas semi-dirigidas hemos abordados las narrativas docentes sobre la con la prueba estandarizada nacional $\mathrm{SIMCE}^{2}$ y el contexto y experiencia de las familias en este barrio pobre. Así, podemos distinguir dos realidades en torno a la justicia. Por un lado, la eterna promesa de entrada al progreso social mediante la formación y acceso al empleo; por otro una justicia de corto plazo que se traduce en asumir las condiciones de pobreza de los alumnos y entregar un apoyo socio-afectivo. El peso del contexto sitúa a muchos docentes en posturas críticas en relación al sistema educativo. Señalando que la vida en la periferia

\footnotetext{
${ }^{1}$ Para profundizar diremos que el polo marginal se refiere al contingente de mano de obra que queda fuera del mercado del trabajo, tanto en los niveles de la economía dominantes, los sectores de extracción de recursos naturales; agricultura, financiero; en el norte y en el sur global. Del mismo modo, los niveles económicos intermedios no pueden absorber a quienes salen del mercado del trabajo, debiendo éstos, situarse en las llamadas economías de subsistencia y de servicio; entrando en dinámicas de precarización laboral y marginalización social (Quijano, 1968; 2000).

${ }^{2}$ SIMCE: Sistema de Medición de la Calidad de la Educación, test que mide los conocimientos en lectura, escritura y matemática (Además de Historia, Ciencias Naturales, actualmente, inglés y educación física). Esta prueba, de carácter nacional, se efectúa en $2^{\circ}, 4^{\circ}, 6^{\circ}$ y $8^{\circ}$ año de educación básica; y en II y IV año de enseñanza media. El SIMCE se inició en 1988, y es el sucesor de la prueba PER (Programa de Evaluación del Rendimiento Escolar), el cual fue aplicado por la Universidad Católica. El SIMCE pasó a ser administrado por el Ministerio de Educación a partir de 1990 (Meckes, 2003).
} 
no permite aplicar a cien por cien la política educativa chilena, pensada desde el centro económico del país. De este modo, el polo marginal de la economía debe ser comprendido según sus propias lógicas, comprendiendo la diversidad de roles y estados que acontecen. En otras palabras, se trata de situarse en la exterioridad (Dussel, 1996) del sistema para comprender y hacer justicia a quienes habitan fuera de los muros del centro cívico.

Esta contribución comenzará por revisar brevemente el concepto de polo marginal, luego, la revisión de la literatura sobre escolarización en contextos de pobreza en Chile; las contradicciones entre lo público-privado. A continuación, ofrecemos el método y los resultados de nuestra investigación; finalizando con la discusión final.

\section{Revisión de la literatura: Polo marginal}

En la reflexión de Aníbal Quijano, la composición de los niveles sociales no se compone de un binario, es decir, dos capas sociales, los integrados y los marginales. Se trata de desequilibrios económicos simultáneos en la gran urbe. En una misma comuna periférica, podemos encontrar barrios con distintos niveles de vida. Se establece a escala micro, una zona integrada y otra marginal. No se trata de un binario centro-periferia; norte-sur global, sino que de un paquete enredado de relaciones desequilibradas que transforman al orden del siglo XIX que construyeron a las sociedades latinoamericanas: periferias urbanas planificadas según una tipología de razas y económica; En la hora actual, con un mercado diversificado, la composición de los barrios experimenta la mezcla socioeconómica difusa o llamada con segregación aventajada o con mixtura social (Ortiz, 2018).

La característica de estos sectores marginados es su pertenencia al sistema. No son expulsados ni reincorporados del sistema bajo los ciclos de crecimiento o de contracción (Quijano, 1968, 2014), sino que nunca han entrado en dichos movimientos.

Si observamos la composición socioeconómica de la Región Metropolitana ${ }^{3}$, apreciaremos cómo los distintos quintiles de la población se encuentran entremezclados en una comuna (con la salvedad de las comunas más ricas). Para Quijano, los desequilibrios en los niveles del sistema es el síntoma de la pauperización social. De este modo, lo que para la escuela austriaca ${ }^{4}$ y Friedman ${ }^{5}$ es el progreso mismo (un empleo de los años noventa dejó de existir o disminuyó notablemente por causa de la tecnología); Sin embargo, no se trata

\footnotetext{
${ }^{3}$ La Región Metropolitana de Santiago. Es una de las quince regiones en las que se encuentra dividido Chile. Es la única región mediterránea y la más pequeña del país. Según el Censo 2017 la población alcanzaba los 7.112 .808 habitantes y se divide administrantemente en seis provincias y 52 comunas (www.ecured.com y www.bcn.cl).

${ }^{4}$ Para una mejor comprensión, ver la definición de Fontaine (1983): "La llamada Escuela Austríaca es una de las ramas más importantes del pensamiento económico y social europeo de nuestro tiempo. Su fundador fue Carl Menger (1840-1921) quien, junto a Jevons y Walras, formuló la teoría de la utilidad marginal del valor o teoría subjetiva del valor económico de los bienes, que marcó un corte con la escuela clásica de Adam Smith, Ricardo y otros, y el comienzo de la escuela neoclásica”.

5 "Milton Friedman: destacado profesor de la Universidad de Chicago, ganador del premio Nobel de Economía en 1976 y creador -junto a Friedrich von Hayek- de la prestigiosa Mont Pelerin Society en 1947. Sin duda, un verdadero liberal, cuyas ideas han ejercido una influencia fundamental en materia económica." (Soto, 2012).
} 
solamente de esta causa, las necesidades del capital hacen que el empleo seguro en un sector; al cabo de unos años, se difumine notoriamente

Dentro de la noción de polo marginal, aparece la crítica a la justicia en tanto acceso al progreso. En el caso de los sectores marginales, el acceso a la escuela, la permanencia y término de la escolaridad se convierten en los primeros logros que permiten superar la etapa de pobreza. A partir de este hito, obtener diplomas, salir de estos barrios, aspirar a un mejor nivel de vida serán los logros más visibles. Las ideas de igualitarismo distribución y/o focalización para nivelar las condiciones; y, el mérito como condición de posibilidad del logro, ponen de manifiesto un discurso y manera de plantear la escolarización en los barrios pobres: la solución para salir de la pobreza es el progreso. Por lo tanto, la justicia está enmarcada por la adopción de la manera de vivir modernacapitalista con perspectiva de aumento del nivel socio-económico (Peña, 2017). En términos de la escolarización esto significa que a mayor nivel educativo, mayores perspectivas de vida asomarán; las necesidades pasarán de las primarias a unas más sofisticadas y el individualismo que propone el mérito dejará de lado formas de vida comunitarias, tanto en los núcleos familiares como barriales que caracterizan a los barrios marginales chilenos, de todas maneras, la entrada a nuevos estadios del sistema se realizan en tiempos distintos, desde los estratos de mayores ingresos hasta las capas más pobres, casi como un pirámide (Acevedo y Javalquinto, 2017). Aníbal Quijano sostiene que tal progreso no puede sino basarse en una marginalización estructural, es decir, más allá de las capas pobres, aparece una multitud humana que simplemente está fuera (Quijano, 1968; 2014), incluso de aquella pobreza que posee un acceso al empleo precario: El llamado polo marginal es aquel que vive permanentemente en la desocupación y marginalidad socioespacial, toda vez que el mercado laboral no necesita de todo el mundo para producir mercancías para el mercado global. Así la justicia por la vía del progreso, en el caso de la escolarización, integrar a los más pobres y excluidos en las aulas, terminará por colisionar con el desempleo estructural y de larga duración.

Una aproximación de la noción de polo marginal con la justicia social se expresa en el reconocimiento del otro (Murillo y Hernández, 2011). Mediante la diferencia, las barreras y dificultades que experimentan distintos grupos de la sociedad pueden ser superadas. En tal sentido, cobra importancia la idea de exterioridad planteada por Enrique Dussel (1996) y su planteamiento del otro: hacer justicia con el pobre, con la viuda y con el extranjero. Así tanto la justicia igualitaria como el mérito personal tienen una superación por la vía de cuestionarse para dónde apunta la justicia: cpara subirse al tren del progreso? Es decir, para acceder a la modernidad capitalista (Peña, 2017). La categoría exterioridad de Dussel tiene correlato en la noción de polo marginal evocada por Quijano, en tanto la justicia dentro del sistema no alcanza para todos. Tal sentencia se debe a que las necesidades que se expresan dentro del mercado se concentran en aquellas que son mayormente difundidas y rentables, por sobre las que buscan preservar la vida de todas y todos (Dussel, 1996). En el caso de la escolarización, mecanismos de financiamiento en base al resultado conseguido; mediciones estandarizadas, rankings de escuelas no hacen más que promover el triunfo de algunos sobre la base del ocultamiento del otro. 


\section{La escolarización en la marginalidad urbana de Santiago, ¿educar en una cultura de la pobreza?}

Siguiendo con la idea de conexión entre sectores marginalizados con el centro urbano de poder, aparecen las instituciones de la sociedad que conectan a las personas en el espacio social. Así, por ejemplo, la primera ley de instrucción primaria planteaba la necesidad de instruir en los saberes considerados esenciales para la nación ${ }^{6}$; si damos un salto en el tiempo, la segunda ley de instrucción primaria plantea con mayor ahínco la instrucción de vastos sectores. La llegada de los gobiernos radicales aparece como un punto de partida de aproximadamente tres décadas de ampliación de la escuela a sectores aun marginalizados. La reforma educativa de Frei Montalva ${ }^{7}$ se abocó en la formación de profesores, la construcción de escuelas, la entrega de material de estudios; tarea que toma Allende y buscó profundizar con la escuela nacional unificada. Pinochet platea en un inicio el volver a los valores occidentales-cristianos, heredados por la llamada chilenidad (Declaración de principios de la Junta Militar, 1974). Luego, el proyecto de los Chicago boys transforma esta primera idea con la masificación de la escolaridad por medio de la libertad de enseñanza (De Castro Spíkula y Méndez, 1992; Jofré, 1988). Con el fin de la dictadura, la concertación busca concluir con la etapa de escolarización o cobertura y apuntar a la modernización de la educación (Comisión "Brunner" 19948), la búsqueda, una vez conseguida la cobertura, es la calidad. Bien podemos quedarnos con esta simplificación, como un breve intento de línea de tiempo de la educación escolar, que nos permite mostrar que los distintos proyectos de masificación de la escuela han establecido un puente de contacto del centro urbano poder con los sectores marginalizados de la población. Desde la instrucción del indio (Lengua castellana, cálculo y catecismo) hasta la escolarización en un gueto del sur de la Región Metropolitana, buscando que tal escuela se supere en sus resultados académicos nacionales, la cuestión (social) que emerge se refiere a la formación integral de un sujeto considerado carente de tales virtudes emanadas de la escuela.

El modelo de escuela privada ideada en Chile muestra que el servicio educativo es administrado y, por ende, provisto por sostenedores educativos privados (los cuales pueden ser personas naturales o jurídicas). A partir de la reforma educativa de los años

\footnotetext{
${ }^{6}$ El vínculo entre nación e instrucción nacional ha sido explicado por Lira (2010) de la siguiente manera: "la comunidad nacional era responsable de asegurar que una mínima dotación de bienes culturales, esenciales para participar de la cosa pública, llegara al conjunto de quienes, a futuro, estaban llamados a ejercer la ciudadanía”.

7 Se trató de la reforma educacional del gobierno de Eduardo Frei Montalva de 1965 y que proponía: "1. La responsabilidad socio-cultural de la educación es promover el desarrollo integral espiritual y material de los individuos, incorporándolos a la vida de la nación, introducir valores democráticos y servir al desarrollo económico de la nación. 2. Preparación para la vida productiva, es decir, la educación debe impartir las destrezas necesarias para una efectiva incorporación al mercado laboral. 3. Acceso a la educación como un derecho humano, es decir, la educación debe hacerse disponible a todos los ciudadanos tanto como sean sus habilidades. 4. La educación para la vida, es decir, la educación no debe ser confinada sólo al período de educación formal durante la niñez y adolescencia, sino que se la debe hacer disponible a toda la población, a través de programas educacionales formales y no formales para adultos sin barreras de edad”. (Biblioteca del Congreso Nacional, 2013).

${ }^{8}$ Comisión asesora para la reforma educativa convocada por el presidente Eduardo Frei Ruiz-Tagle en 1997.
} 
ochenta, el número de escuelas particulares subvencionadas ha crecido significativamente 9 . Con especial atención en abrir centros educativos en barrios marginales.

\section{Método}

Nuestra investigación ha consistido en un estudio de caso de tipo descriptivo, en el cual lo orientamos en explorar las eventuales diferencias que pudiesen existir entre la escuela particular subvencionada y la escuela municipal en la escolarización de niños de sectores marginalizados. Una vez determinada esta situación hemos buscado la prueba crítica (Díaz De Salas, Mendoza y Porras, 2011) que nos permitiese aseverar o destacar las diferencias entre estos tipos de establecimientos.

Mediante la aplicación de entrevistas semi-dirigidas a dos profesores por cada establecimiento municipal y escuela particular subvencionado en tres comunas periféricas de Santiago de Chile, a saber, Recoleta, Quilicura y La Pintana ${ }^{10}$. La razón para hacer tal distinción metodológica es observar el funcionamiento de ambos tipos de establecimientos dentro de un sistema educativo de cuasi-mercados (Hernández-Pedreño, 2010). En este caso, hemos buscado la respuesta a la pregunta: ${ }_{¿}$ Existen diferencias en la calidad en las escuelas particulares, en relación a las escuelas públicas seleccionadas?

Las preguntas abordaron diversas temáticas sobre el establecimiento: nos focalizamos en cuatro grandes categorías pre-establecidas: liderazgo, calidad, relación escuela - población y elección de la escuela (Rodríguez Molina y Gairín, 2017). De estos temas, hemos seleccionado el rol de la prueba estandarizada nacional SIMCE; más la relación de las escuelas con los barrios periféricos, las condiciones socio-económicas de los alumnos y sus familias. Las respuestas de los profesores tomaron siempre como referencia el contexto y las experiencias de vida de los alumnos y sus familias en la población. Las visitas a las escuelas se realizaron entre los años 2017-2018. El análisis de las entrevistas lo realizamos mediante la codificación axial de diversos temas que resurgían a cada categoría, para esto, nos hemos apoyado en el programa Nvivo $11^{11}$; luego de esto, hemos revisado las categorías pre-establecidas, unificando los códigos, de manera tal de obtener nuevas categorías de análisis, las cuales. Para este artículo, hemos seleccionado las siguientes categorías y subcategorías:

- Categoría 1: El rendimiento académico de los alumnos y el SIMCE dentro de las prioridades de los profesores

\footnotetext{
${ }^{9} \mathrm{El}$ número de establecimientos particulares subvencionados es de 5.665; mientras que el de los establecimientos municipales es de 4.925, considerando, en ambos casos, establecimientos reconocidos oficialmente por el estado de educación parvularia, primaria y secundaria (Fuente: Estadísticas de la educación, 2018, Ministerio de Educación, Chile).

${ }^{10}$ Recoleta es una comuna limítrofe con la ciudad de Santiago, se trató de la primera periferia fundada por los conquistadores españoles; Quilicura se encuentra en el extremo norte de la región. Ahí, podemos encontrar blocks y departamentos sociales, fruto de la política habitacional de los gobiernos de la concertación; El Castillo, es un suburbio del sur de Santiago, dentro de la comuna de La Pintana: formada por grupos erradicados desde las zonas más ricas de la región. Medida tomada por la dictadura militar que obligó a los pobladores a instalarse en comunas periféricas.

${ }^{11}$ NVivo es un software para la investigación con métodos cualitativos y mixtos.
} 
Subcategoría A: Escuelas particulares subvencionadas, normatividad y SIMCE

Subcategoría B: Escuelas municipales, SIMCE, trabajo docente y su relación con los alumnos.

- Categoría 2: Representaciones de la población como espacios de conflicto

$\checkmark$ Subcategoría A: Escuelas particulares subvencionadas, espacios resguardados frente al contexto

$\checkmark$ Subcategoría B: Escuelas municipales: resignación frente al contexto familiar

Para facilitar la identificación de los entrevistados, hemos dispuesto estas etiquetas que distinguen a cada uno: Profesor + materia que enseña (Lenguaje o matemática) + PS (particular Subvencionado) o MUN (Municipal) + guion bajo + REC (Recoleta) o (Quili (Quilicura) o PINT (la Pintana).

Estas identificaciones se encontrarán entre paréntesis, al final de cada párrafo de las entrevistas, los cuales están con letrea cursiva. Algunos ejemplos de identificación: Profesor lenguaje, PS_Rec, Profesora matemática.

\title{
5. Resultados
}

\subsection{Categoría 1: El rendimiento académico de los alumnos y el SIMCE dentro de las prioridades de los profesores}

\subsubsection{Subcategoría A: Escuelas particulares subvencionadas, normatividad y SIMCE}

En términos generales, el SIMCE no es considerado como un aspecto indispensable en la escuela. En las escuelas particulares subvencionadas, una primera reacción una profesora nos declara:

\begin{abstract}
Acá, el SIMCE no es tema, de hecho, por eso nos criticaron del ministerio: ustedes son un colegio, no un hogar yo discrepo de eso, creo que desde este aspecto se pueden lograr mucho más que desde el conductismo y la rigurosidad de estar parados frente a ellos y dejando de lado que el niño vienen con una carga importante que es necesario solucionar o hacer que el niño lo sepa sobrellevar de buena manera. (Profesora matemática, PS_Rec)
\end{abstract}

En el caso de este establecimiento, las prácticas escolares no se encuentran en correlación con el SIMCE, ubicándose éste en un lugar inferior dentro de las prioridades. La crítica del Ministerio de Educación sobre el papel que debe desempeñar la escuela revela que existen dos miradas dispares entre ambas instituciones.

Si el SIMCE no es la prioridad según los actores escolares, ¿Por qué entonces darle importancia? ¿La mera obligación que ley demanda? Una profesora nos dice:

[El SIMCE] no es prioridad para el colegio, pero si hay preocupación ya que los establecimientos reciben beneficios por tener buenos resultados en el SIMCE y también es un punto que se considera para lograr la excelencia académica. (Profesora matemática, PS_Pint)

Los beneficios materiales y simbólicos que el SIMCE otorga resulta atrayente o no una condición que se deba obviar. La excelencia académica se refiere al reconocimiento que una escuela recibe por parte del Ministerio de educación, en términos de efectividad, 
rendimiento sostenido o superación, igualdad de oportunidades, iniciativa, entre otros indicadores medibles.

Sobre este punto, las escuelas particulares subvencionadas nos mostraron que los docentes emplean distintos planes para la preparación de este examen.

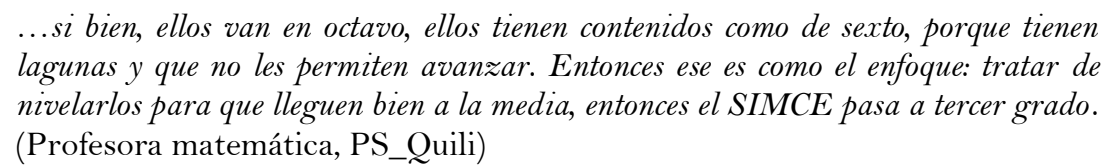

La nivelación o reforzamiento de las habilidades medidas por el SIMCE ocupa un lugar dentro del trabajo en la sala de clases.

5.1.2. Subcategoría B: Escuelas municipales, SIMCE, trabajo docente y su relación con los alumnos.

$\mathrm{Al}$ igual que en el caso de las escuelas particulares subvencionadas, los establecimientos públicos deben ocuparse de esta evaluación. El trabajo dedicado no se diferencia tanto. Una profesora lo señala:

preparamos guías, cada quince días tenemos planificadas guías de lenguaje entonces,
con análisis, sin análisis, partiendo ahora por textos cortos, porque el SIMCE vienen
con textos largos, pero no se les mete miedo que les va a ir mal, tratamos que los
chiquillos puedan entender lo que están haciendo y es importante porque en los colegios
municipales el SIMCE es el que sirve pa' subir un poco como lo miden afuera en las
municipalidades. (Profesora lenguaje, Mun_Rec)

La finalidad del SIMCE, sin embargo, varía, puesto que el nivel académico de los alumnos es juzgado como bajo. Ante esto, los objetivos son adaptados a la medida del alumnado:

Que sepan que tienen que estar sentados dos horas y cuarto, dos horas y media, no me acuerdo cuánto es. (Profesora lenguaje, Mun_Rec)

La duración del SIMCE no se presenta como un tema accesorio, por el contrario, la cantidad de horas repercute en el rendimiento de cada alumno. Este aspecto pasa a tener relevancia y debe ser atendido.

Otro elemento que aparece en las entrevistas se refiere a la adaptación del currículum en contextos vulnerables: la posición mayormente sostenida, dice relación con el nivel académico del alumnado, considerado bajo por parte de los docentes.

Yo a ellos no les puedo pasar los mismos contenidos que les pasan en los otros colegios porque yo tengo muchos niños que no saben multiplicar. (Profesora matemática, Mun_Pint)

El factor tiempo y el factor nivel académico de los estudiantes contribuyen a acrecentar la carga horaria del profesor. De todas maneras, estos factores aportan una mirada distinta sobre la calidad de la educación: por calidad no se entenderá exclusivamente el logro de estándares prediseñados y puntajes SIMCE elevados, el giro que torna la noción apunta principalmente a nivelar y a velar por otros valores que no se encuentran necesariamente en evaluaciones estandarizadas.

\subsection{Categoría 2: Representaciones de la población como espacios de conflicto}

La población fue identificada como un lugar depositario de la vulnerabilidad social en la que los niños de las escuelas visitadas viven, se desenvuelven. En las escuelas visitadas, la apreciación de este espacio de la ciudad es mayoritariamente compartida. 
5.2.1. Subcategoría A: Escuelas particulares subvencionadas, espacios resguardados frente al contexto

Las diferentes vivencias que acontecen en la población son integradas en la caracterización general que se tiene. Hechos delictuales y de violencia entregan una opinión sobre la población, caracterizándola negativamente. Una profesora nos introduce:

\section{Los niños vienen de lugares que son muy malos, la conocida Parinacota que sale en las noticias, entonces vienen de esos sectores también. (Profesora matemática, PS_Quili)}

El reconocimiento de un barrio peligroso, llamado en Chile la población, contiene una carga valorativa importante. La utilización del articulo $L a$, el cual indica la población, más el nombre del barrio (aunque en varios casos posean otro nombre) marca claramente el lugar. Este punto se refuerza con la calificación que el entrevistado da de este sitio: "lugares que son muy malos".

Esta opinión es refrendada por otra profesora:

Hay algunas partes que son bastante complicadas La Parinacota, La San Luís, hacia allá entonces en un momento tuve problemas con mis alumnas que el furgón, fue operada y necesitaba un furgón, y los furgones no iban hacia donde vivía ella porque a los tíos del furgón les daba miedo ir a buscarla. (Profesora matemática, PS_Quili)

Este caso particular evidencia que un factor importante opera en la valoración de la población como lugar de conflicto. El miedo experimentado por una persona vinculada a la escuela construye una visión negativa sobre la población.

La población caracterizada como un lugar malo y que provoca miedo de ingresar en ella obedece a la identificación de prácticas de marginalidad que los entrevistados entregaron. El detalle de éstas fue mencionado por un profesor:

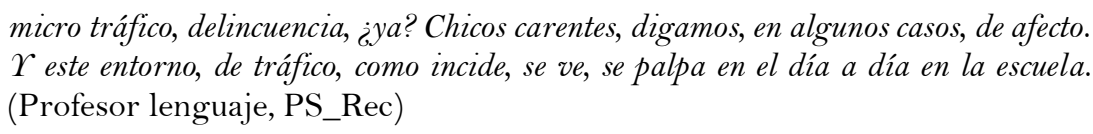

La droga, la delincuencia y las carencias afectivas son tres categorías que fueron mencionadas durante las entrevistas. Estos factores se evidencian en la cotidianeidad de la escuela, aunque no nos fue posible indagar más sobre este punto. La convivencia escolar en las escuelas particulares subvencionadas visitadas podría apreciarse mejor solidificada que en las escuelas municipales.

Este punto nos parece de mucha importancia. La pregunta que se abre dice relación con las reales influencias que la población ejerce sobre el comportamiento de los niños. Las escuelas particulares subvencionadas lograrían, de mejor manera, que las municipales una asimilación de su cultura escolar en las familias que se escolarizan.

$$
\begin{aligned}
& \text { Lo que pasa es que a esta escuela llegan todos los niños que, por decirlo de alguna } \\
& \text { manera, ningún otro colegio quiere. (Profesora matemática, PS_Quili) }
\end{aligned}
$$

En este caso, esta escuela no selecciona a sus alumnos y orienta su proyecto educativo en la integración de niños en situación de vulnerabilidad social, toda vez que otros establecimientos del sector no están dispuestos a hacerlo. De todos modos, el enfoque dado por esta escuela se inscribe más en la ayuda material a familias con pocos recursos (entrega de material escolar, uniforme, alimentación) más que en el análisis y tratamiento de situaciones conflictivas. 
La vida en la población parece tener consecuencias bastante difíciles para las familias que frecuentan la escuela. Aunque la composición del alumnado de las escuelas particulares subvencionadas no proviene de exclusivamente de estos sectores, existe una mayor mixtura de alumnos. Una profesora nos explica:

\begin{abstract}
Niños que vienen del Palmarés, que acá son colegios más reconocidos entre comillas, con familias que tienen más recursos, y tenemos niños que vienen de la Parinacota, del Valle de la luna, de la San Enrique, entonces hay como una diversidad, pero al final, todos tienen las mismas necesidades. (Profesora lenguaje, PS_Quili)
\end{abstract}

Este párrafo expresa que existe una diversidad socioeconómica importante en la escuela particular subvencionada, viniendo alumnos de distintos lugares y con distintos medios económicos, sin embargo, las carencias son percibidas, por los profesores, que, en el caso de la población, se refuerzan y se justifican más claramente como el producto de la cultura que se construye en estos sitios.

En las escuelas particulares subvencionadas que visitamos existe una constatación sobre la población como espacio de conflicto. La caracterización de estos lugares no dista mucho de aquel que tiene la opinión pública. La presencia de delincuencia, tráfico de droga es observada como un universo desconocido en el cual es mejor no involucrarse demasiado dado la sensación de temor que acontece. De esta forma, la escuela pasaría a convertirse en un pórtico que separa la población con todas sus características negativas, del mundo social mayor que la escuela busca desarrollar.

\title{
5.2.2. Subcategoría B: Escuelas municipales: resignación frente al contexto familiar
}

En el caso de las escuelas municipales, las entrevistas nos entregaron más información sobre las poblaciones aledañas, de donde provienen las familias que frecuentan estos establecimientos. La composición del alumnado que proviene de estos sectores es superior al caso de la escuela particular subvencionada.

Los entrevistados caracterizaron los barrios cercanos a los establecimientos municipales, evidenciando una serie de problemas sociales y de actitudes adoptadas por los alumnos y causadas esencialmente por la crianza de éstos en un entorno complicado. Un docente nos dice:

\footnotetext{
El entorno del colegio es bien complicado: tenemos poblaciones acá en Quilicura que son bastantes complejas: acá al frente del colegio hay una de las poblaciones que vienen la mayoría de ellos de ahí, que son poblaciones que encontramos tráfico, prostitución y eso es familiares de los mismos alumnos que nosotros tenemos en el colegio. (Profesora lenguaje, Mun_Quili)
}

La mayoría de las entrevistas caracterizaron los focos de violencia bajo los adjetivos de complicado-a o complejo-a, escondiendo, por un lado, una descripción más detallada de cada hecho; y por otro lado, entregando una nueva significación a estos adjetivos. De este modo, cuando hablamos de un barrio complicado o complejo, lo entenderemos como violento, con presencia de narcotráfico, donde se suceden enfrentamientos armados, además de prostitución.

A continuación, un docente nos recuerda que es preciso tener siempre presente el origen de los estudiantes:

Son todos de la población, no hay que olvidar nunca eso, que, pese a todas sus deprivaciones, llegan a acá. (Profesor matemática, Mun_Rec) 
Esta primera mirada expone que el peso de la población en la vida de los alumnos que llegan a la escuela es importante y que no debe dejarse de lado este elemento, toda vez que condiciona la vida dentro de la escuela.

El escenario se encuentra marcado por lo tanto por la cultura que la población posee, a este respecto. Una docente recalca:

$$
\begin{aligned}
& \text { Aquí en las poblaciones acá' cercanas, mucho tráfico, balaceras, o sea es una realidad } \\
& \text { complicadita. (Profesora matemática, Mun_Pint) }
\end{aligned}
$$

Aparecen situaciones vinculadas a la delincuencia y tráfico de drogas. Estos fenómenos se vislumbran frecuentes en el diario vivir de estos barrios y su influencia, por ende, es significativa para las escuelas municipales.

La forma visible de palpar la marginalidad de las poblaciones, descrita en las entrevistas, se observa en elementos que son portados por ciertos alumnos.

En el sector donde vivo aquí, tú sabes que las escuelas municipales, los cabros que van con cuchillas... a mi el año pasado me tocó que un niño viniera con pistola, con cuchillo, y tú tienes que ponerte serio. (Profesora lenguaje, Mun_Pint)

Esta idea fue replicada en varias entrevistas que realizamos. El uso de armas forma parte de la cultura en muchos espacios. La imagen de las escuelas municipales, en especial, como establecimientos que reciben a alumnos que incurren en actos delictivos se plasma como una característica importante.

Las entrevistas evidenciaron que estos casos se explican por las dinámicas que se viven en la población, generando formas de resolución de conflictos que implican el uso de armas; por lo tanto, contienen una aceptación que se refleja por el grado de normalidad. Esto fue destacado durante las entrevistas. Otra profesora nos señala:

Entonces la forma de cómo te lo cuentan es como no profe, yo el otro día estaba en la panadería y había unos cabros con pistola y yo saqué mi pistola y después llegaron los pacos y nos subimos al techo y andaba todo tajeado aquí. (Profesora matemática, Mun_Pint)

Esta descripción da cuenta de esta normalidad de las historias que escuchan los docentes. En este caso, el relato de un asalto a mano armada y la posterior escapatoria de menores (suponemos que lo son toda vez que la entrevistada habla de unos cabros). Se parecía en las escuelas municipales una convivencia mayor con este tipo de acontecimientos, lo cual refleja que la escuela municipal no llega al nivel de protección o aislamiento que poseen las escuelas particulares subvencionadas, en relación al contexto de marginalidad del barrio.

De todas maneras, el trabajo de mejora del clima escolar como cimiento del proyecto educativo aparece justificado de manera más amplia. La erradicación de formas culturales asociadas a la marginalidad social toma mayor relevancia en las escuelas municipales.

Ellos son uno más dentro de una heterogeneidad, aquí no hay problemas, como te podría explicar, como se le denomina, el flayterismo ${ }^{12}$, ¿Comprendes el concepto? aquí no se ve eso. (Profesor matemática, Mun_Rec)

${ }_{12}$ Flaite o flayte; Se ha definido de muchas maneras, entre las cuales seleccionamos: "persona de clase social baja y comportamiento extravagante, que es relacionada generalmente con el mundo delictual”, según la definición del (Diccionario de uso del español de Chile, citado por Rojas, 2015). Adaptación del inglés con la palabra "Flighter" que significa "Volador" esto se usaba para llamar a 
La escuela busca crear un patrón común basado en el establecimiento de una convivencia escolar. Esta se expresa en planes y medidas concretas (los mencionados recreos entretenidos, por ejemplo) que intentan contrarrestar culturas de la población, que operarían, según el discurso de los entrevistados, como contra-culturas. El flayterismo que nos señala este entrevistado, se refiere a variadas formas de expresión, a saber, lenguaje, vestimenta, rituales, estilos musicales, entre otros, propios de la cultura de la calle. Aquí aparece una contradicción entre el sujeto que se escolariza (de la población) y el perfil de alumno que se busca (misión del establecimiento). La idea de la escuela como el pórtico o pasaje de una sub-cultura a una cultura más avanzada, se evidenciaría como trasfondo de las entrevistas.

$\mathrm{Al}$ igual que en el caso de las escuelas particulares subvencionadas, las entrevistas en las escuelas municipales ubicaron a las poblaciones en una órbita diferente al de otros sectores de la ciudad, explicitándolas como un espacio desconocidos, en los cuales es difícil involucrarse, producto de la fuerte carga de violencia que en ellas se encierra. Una profesora nos cuenta una vivencia: Donde yo vivo, por aquí también, viste, las calles no tienen ni nombre porque así no
pillan a nadie. (Profesora lenguaje, Mun_Rec)

Esta práctica propia del narcotráfico expresa el conflicto permanente que los entrevistados atribuyen a la población. El ocultamiento de calles para así despistar a la policía, sumado a que muchos de estos barrios poseen una urbanización bastante irregular, toda vez que muchas de las poblaciones fueron el producto de tomas de terreno en décadas pasadas, generan un diseño complejo, de difícil determinación. Este tramado de las calles concurre en la caracterización de lugar ajeno a la realidad que se busca construir en la escuela.

Por último, una carencia importante de las familias que viven en poblaciones aledañas a las escuelas, es la falta de alimentación que muchos niños experimentan diariamente. En este caso, la escuela emerge como lugar donde se puede satisfacer esta necesidad. Un profesor nos dice:

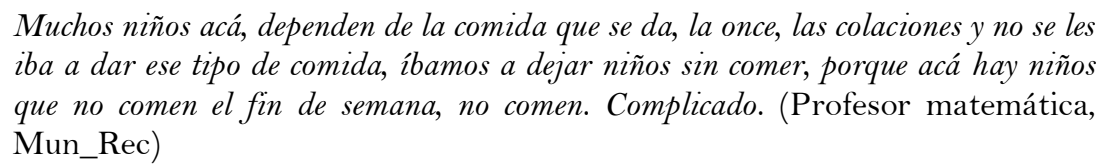

Este punto fue destacado en esta entrevista y muestra que la escuela incorpora en su esencia una labor social que va más allá del carácter académico.

En las entrevistas realizadas, hemos constatado que la población es identificada como un espacio de conflicto, donde muchas prácticas asociadas a la delincuencia y tráfico de drogas marcan fuertemente el discurso de los entrevistados. En las escuelas particulares subvencionadas, las entrevistas mostraron que existe una distancia entre las escuelas y los barrios: si bien, se reconoce la peligrosidad de ciertos lugares y momentos del día, no se relataron hechos de este tipo dentro de los establecimientos, lo que constituiría una zona de protección para las familias que frecuentan estas escuelas. Del lado de las escuelas

las personas que consumían Drogas...(https://diccionariochileno.cl/term/Flaite). Flaite vendría de un modelo de zapatillas llamado Air Flight desarrollado por la marca Nike. Los modelos piratas se llamaban Flight Airs, de ahí derivó en "flaiters" y su uso se expandió para referirse a los jóvenes que las usaban (El Definido, Conoce el origen de algunos de nuestros chilenismos, 17 de septiembre de 2013, citado por Rojas, 2015). 
municipales, las situaciones de delincuencia y narcotráfico fueron descritas con mayor detalle, revelando que algunas de estas ocurren dentro del establecimiento. El porte de armas principalmente es mencionado. No se mencionaron otras organizaciones de estos barrios que también se encuentran (Juntas de vecinos, asociaciones y talleres culturales, iglesias, grupos políticos, clubs de fútbol y otros espacios de educación no formal). Por último, se reconoció en una entrevista que la alimentación que se ofrece en las escuelas es valorada y atiende a una carencia importante en familias de bajos recursos.

\section{Discusión y conclusiones}

Las entrevistas realizadas a profesores en seis escuelas de tres comunas periféricas de Santiago nos entregaron importantes clarificaciones sobre las dificultades experimentadas por niños y sus familias de poblaciones marginalizadas: violencia física, verbal, narcotráfico, trabajo infantil, reflejaron en muchos casos una resignación sobre una realidad que parece no cambiar. La escuela fue caracterizada como zona de protección, seguridad; y también como punto de encuentro con las familias. Apreciamos poca integración con el barrio. Las realidades son distintas de una escuela a otra y dan cuenta de la noción de polo marginal de Aníbal Quijano, el cual se presenta como una heterogeneidad estructural: múltiples casos e historias de vida, casi individuales, pero agrupados en un gran sector de la población chilena, sobrepoblada o hacinada en torno al centro urbano. ¿Son pobres desde el punto de vista material? No necesariamente; el SIMCE es bajo, a veces; pero, del mismo modo, podemos encontrar en una persona, ambas situaciones, es por eso que Quijano le llama estructural en otras palabras, la pobreza, precariedad, violencia, trayectorias escolares interrumpidas, bajos resultados son algunas categorías dentro de una estructura fija, constante, que se encuentra lejos del centro.

En relación a la justicia, aparecen dos imperativos. Por un lado, el reconocimiento entre alumnos y profesores, la protección ante los peligros, el compromiso que muchos docentes y apoderados muestran, y que vimos presencialmente; Por otro lado, las obligaciones que el sistema escolar manda: bajo el paradigma de la excelencia, la calidad de la educación sería el vector de movilidad social, produciéndose una justicia social en base a los méritos individuales (Tenti Fanfani, 2003). No obstante, la prueba SIMCE genera una tensión por la cual deben transitar los alumnos, profesores y las escuelas: básicamente, cumplir con la exigencia del paradigma de la educación de calidad en condiciones complejas. Así, en las escuelas particulares subvencionadas, existe un margen mayor que permite dar la evaluación estandarizada SIMCE una importancia distinta; en las escuelas municipales, asoman estrategias que buscan obtener un resultado digno en la medida de lo posible. De este modo, el problema del SIMCE no es tanto un resultado o la búsqueda de la excelencia, sino cómo enfrentar esta prueba sin bajar el promedio, pero teniendo en cuenta las dificultades sociales y de los aprendizajes de los alumnos.

Antes este panorama, nos parece importante reflexionar sobre el espacio periférico y el concepto de pobreza. Hemos hablado de forma fuerte sobre la marginalidad, el sujeto pobre, la violencia, el uso de identificaciones tales como delincuente. El uso de estos términos lo hemos planteado desde la negatividad como hilo conductor en este análisis: lo que es despreciado por la modernidad (chilena), los saberes (jergas, imaginarios, construcciones sociales periféricas, organizaciones) despreciados por el currículum y que, para nosotros, representan realidades, ante todo. La negatividad del sistema, todo (y todos) aquello/s que no entra/n en la realidad que decimos ver y que es producto de la 
materialidad existente: ¿acaso el narcotráfico no es un hijo no reconocido del capitalismo y/o de la privatización? (la racionalidad con la que opera un traficante no difiere mucho de la empleada por un gran empresario, nos referimos en términos de ver el mundo según los activos y pasivos que se pueden obtener; lo que cambia en uno y otro caso son las condiciones de existencia y los medios empleados), cuando mencionamos la noción de exterioridad, nos referimos precisamente al espacio no cubierto total o parcialmente por el orden existente. ¿Quién quiere ir a trabajar en una escuela de La Pintana, Recoleta o Quilicura? Esta pregunta más que analizarla como un menosprecio, la planteamos como un constructo mental establecido. La respuesta sería, a nuestro parecer, que muy poca gente querrá ir. Es lejos del centro, queda lejos. Pues bien, ¿Quién define que La Pintana queda lejos? ¿Por qué la representación general casi automática es asociar esta comuna a la lejanía? Podemos formular las mismas preguntas sobre otras representaciones sobre La Pintana. Estas preguntas son formuladas desde el centro, desde la totalidad del sistema: desde este lugar, no solo nacen estas interrogantes, sino que las soluciones (y los problemas) que, digamos, padecen estas comunas.

\section{Agradecimientos}

Este trabajo forma parte de la investigación: "Privatización de la educación en Chile: estudio del caso de la escuela privada en sectores vulnerables". Tesis de doctorado en ciencias de la educación de la Universidad de Ginebra, realizada por Ignacio Reyes Cayul. Financiamiento recibido de Becas Chile. También a la Universidad de Playa Ancha y el apoyo del Ministerio de Educación a través del Plan de Fortalecimiento de Universidades Estatales UPA 1799.

\section{Referencias}

Acevedo, R. y Javalquinto, B. (2017). Banco mundial, ajustes estructurales y escuela de los Chicago boys. Revista Chilena de Economía y Sociedad, 2, 65-71

De Castro Spíkula, S. y Méndez, J. C. (1992). El ladrillo. Bases de la política económica del gobierno militar chileno. Centro de Estudios Públicos

Díaz De Salas, S. y Mendoza Martínez, V. y Porras Morales, C. (2011). Una guía para la elaboración de estudios de caso. Razón y Palabra, 75, art 3.

Dussel, E. (1996). Filosofía de la liberación. Nueva América.

Fontaine, F. (1983). La crítica de la "escuela austríaca" al socialismo. UDP.

Lira, R. (2010). Proyecto de nación y obligatoriedad escolar: Antecedentes y proyecciones a noventa años de promulgada la ley de educación primaria obligatoria. Pensamiento Educativo, $46,45-62$.

Meckes, L. (2003). El SIMCE: Su desarrollo y desafíos actuales. Pensamiento Educativo, 33, 160178.

Murillo, F. J. y Hernández, R. (2011). Hacia un concepto de justicia social. REICE. Revista Iberoamericana sobre Calidad, Eficacia y Cambio en Educación, 9(4), 7-23.

Ortiz, I. (2018). Relación entre la composición social de la comuna y la de las escuelas en la región metropolitana de Santiago. Calidad en la Educación, 48, 166-203.

https://doi.org/10.31619/caledu.n48.483 
Peña, C. (2017). ¿Cuánta desigualdad necesita la democracia? Estudios Públicos, 147, 57-69. https://doi.org/10.38178/cep.vi147.99

Quijano, A. (2011). Colonialidad del poder y clasificación social. Contextualizaciones Latinoamericanas, 3(5), 1-33.

Quijano, A. (1968). Dependencia, cambio social y urbanización en Latinoamérica. En D. Assis (Ed.), Cuestiones y horizontes de la dependencia histórico-estructural a la colonialidad/descolonialidad del poder (pp. 99-128). CLACSO.

Quijano, A. (2000). Colonialidad del poder, eurocentrismo y América Latina. En E. Lander (Comp.), La colonialidad del saber: Eurocentrismo y ciencias sociales. Perspectivas latinoamericanas (pp. 5769). CLACSO.

Rodríguez Molina, G. y Gairín, J. (2017). Influencia de las prácticas de liderazgo pedagógico en las prácticas pedagógicas docentes: caso en Chile de las unidades técnicas pedagógicas. International Journal of Educational Leadership and Management, 5(1), 6-29.

https://doi.org/10.17583/ijelm.2017.2469

Rojas, D. (2015). Flaite: Algunos apuntes etimológicos. Alpha, 40, 193-200. https://doi.org/10.4067/S0718-22012015000100015

Soto, A. (2012). Un legado de libertad. Milton Friedman en Chile. Fundación para el Progreso.

Tenti Fanfani, E. (2003). La escuela y los modos de producción de la hegemonía. FLACSO. https://doi.org/10.17227/01203916.5490

\section{Breve CV del autor}

\section{Ignacio E. Reyes}

Profesor de francés de la Universidad Metropolitana de Ciencias de la Educación desde 2009. Máster en ciencias de la educación en la Universidad de Ginebra y Doctor en Ciencias de la Educación por la misma universidad. Actualmente, investigador postdoctoral en la Universidad de Playa Ancha y docente del Departamento de Arte y Humanidades de la Universidad de Concepción. Mis termas de investigación son: privatización de la educación; migración, ciudadanía y políticas lingüísticas. ORCID ID: https://orcid.org/OOOO-OO01-8494-716X. Email: ignacio.reyes.cayul@gmail.com 